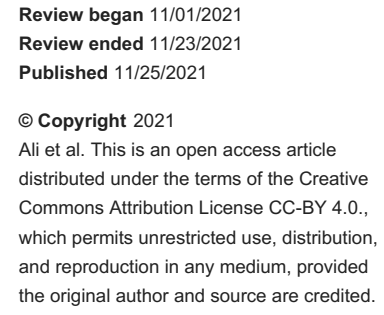

\section{Symptomatic Gallstones in Young Patients Under the Age of 30 Years}

Asif Ali ${ }^{1}$, Sughra Perveen ${ }^{2}$, Imran Khan ${ }^{2}$, Tanweer Ahmed ${ }^{2}$, Ali Nawaz ${ }^{2}$, Abdul Rab ${ }^{2}$

1. Department of Surgery, Jinnah Postgraduate Medical Centre, Karachi, PAK 2. Department of General Surgery, Jinnah Postgraduate Medical Centre, Karachi, PAK

Corresponding author: Asif Ali, aakhowaja09@gmail.com

\section{Abstract \\ Introduction}

Gallstones are deposits of digestive fluid that is hardened into small pellets. Gallstones can be symptomatic or asymptomatic. The present study assessed the sociodemographic and clinical characteristics of patients under the age of 30 years old with symptomatic gallstones.

\section{Methodology}

A prospective, observational study was conducted at Surgical Unit-3, at Jinnah Postgraduate Medical Centre (JPMC), Pakistan, from July 2020 to July 2021. All patients suspected of having gallstone disease underwent ultrasonic examination. A diagnosis of gallstone disease was made if the gallbladder showed a hyperechoic mass casting a posterior acoustic shadow, with a change of position. All of the patients with gallstone disease were hospitalized. The patients were asked about their age, past history (with special emphasis on last pregnancy and years of oral contraceptive use, if any), socioeconomic status, and other demographic data. The patients were treated with a cholecystectomy, either laparoscopic or open. Postcholecystectomy complications, if any, were recorded.

\section{Results}

A total of 210 patients under the age of 30 years were included. The mean age of patients was $25 \pm 3$ years The majority of the population were females, i.e., 170 (81\%). Upon assessing the risk factors, it was found that $31.84 \%$ of the female patients had used oral contraceptives, $32.96 \%$ were pregnant at the time of presentation, $20.48 \%$ had diabetes mellitus, and $27.14 \%$ had a history of gallstones. Furthermore, it was found that about 114 (54.29\%) patients were overweight with a body mass index (BMI) of $25-30 \mathrm{~kg} / \mathrm{m}^{2}$. High triglyceride levels and high cholesterol levels were found in 108 (51.43\%) and 115 (54.76\%) of the patients, respectively, indicating a link between symptomatic gallstones and younger age.

\section{Conclusion}

Our study indicated that young people are prone to symptomatic gallstones. The rate of disease was considerably high among females and in patients with high cholesterol and triglyceride levels and abnormal body mass index. Further exploratory studies are needed to determine the cause of gallstones.

Categories: Gastroenterology, General Surgery, Public Health

Keywords: gallbladder, cholelithiasis, young patients, symptomatology, diabetes mellitus, obesity

\section{Introduction}

Gallstones are deposits of digestive fluid that is hardened into small pellets. Gallstones can be symptomatic or asymptomatic [1]. The size of gallstones varies from grains of sand to golf balls. Gallstone attacks usually cause vomiting, nausea, and pain in the stomach, ribs, and between the patients' shoulders. In some severe cases, it may cause complications such as acute pancreatitis, jaundice, bowel obstruction, and gallbladder carcinoma and may require cholecystectomy $[2,3]$.

Gallstone is a very common disease, and it has been classified into two types: cholesterol stone, which has $>70 \%$ cholesterol content, and pigment stone, which has $<30 \%$ cholesterol content [4]. A study on postoperative patients revealed that the rate of symptomatic cholelithiasis was 3.5\%, with a mean duration of symptoms of around 12 months. They revealed that sudden weight loss was a significant factor that led to the development of cholelithiasis [5]. Females have a higher chance to develop cholelithiasis. This may be due to hormonal and reproductive factors, i.e., oral contraceptives and pregnancy. Another study from India revealed that the incidence of cholelithiasis is $21.76 \%$ among patients who underwent a bariatric procedure [6].

Literature showed that cholelithiasis is strongly related to body mass index (BMI) [7,8]. This may be due to 


\section{Cureus}

increased fat consumption and less physical activity. The prevalence of gallstones is also very common in the upper and middle socioeconomic class due to their dietary habits and obesity. The treatment of gallstone depends upon how many symptoms a patient is showing $[9,10]$. For symptomatic gallstones, surgery is performed to remove gallstones from the gallbladder. However, due to the scarcity of local literature, we cannot ascertain the epidemiology of gallstones in the younger Pakistani population. Thus, the current study was conducted to find the sociodemographic and clinical characteristics of young patients with symptomatic gallstones.

\section{Materials And Methods}

From July 2020 to July 2021, a prospective, descriptive study was conducted at Surgical Unit-3, at Jinnah Postgraduate Medical Centre (JPMC). The ethical committee of JPMC granted approval for this study with the reference number F9-98-IRB-2018-G/28867/JPMC. A total of 210 patients under the age of 30 years, irrespective of gender, with a diagnosed gallstone disease were included in the study group. Informed written consent was obtained from all patients. All those who presented with the symptomatology of biliary colic without any evidence of gallstones were excluded.

All patients suspected of having gallstone disease underwent ultrasonic examination. A fasting period of 12 hours was required prior to the examination, which was carried out in the supine position. A diagnosis of gallstone disease was made if the gallbladder showed a hyperechoic mass casting a posterior acoustic shadow, with a change of position. All of the patients with gallstone disease were hospitalized. Each participant was personally interviewed by trained interviewers.

The patients were asked about their age, past history (with special emphasis on last pregnancy and years of oral contraceptive use, if any), socioeconomic status, and other demographic data. Family histories of gallstones and diabetes mellitus were also determined. Basic data on weight with the patient clothed and height without shoes was documented. Body mass index $\left(\mathrm{kg} / \mathrm{m}^{2}\right)$ was calculated as a measure of obesity. The patients were categorized as normal weight when their BMI is less than 25, overweight when their BMI is 25-30, and obese when their BMI exceeds more than 30.

All patients underwent laboratory analysis for a complete blood picture with reticulocyte count, serum transaminases, bilirubin level, and fasting lipid profile. The patients were treated with a cholecystectomy, either laparoscopic or open. Postcholecystectomy complications, if any, were recorded. Data were recorded and analyzed using the Statistical Package for the Social Sciences (SPSS) version 16.0. Descriptive statistics were calculated for both qualitative and quantitative variables. Mean \pm SD was calculated for demographic data.

\section{Results}

A total of 210 patients under the age of 30 years were included. The mean age of the patients was $24.65 \pm$ 3.25 years. The majority of the population were females, i.e., 170 (80.9\%) (Table 1).

\begin{tabular}{|l|l|}
\hline Characteristics & $\mathbf{n}(\%)$ \\
\hline Gender & $40(19.04 \%)$ \\
\hline Male & $170(80.9 \%)$ \\
\hline Age & $24.65 \pm 3.254$ \\
\hline Socioeconomic status & \\
\hline Lower & $33(15.71 \%)$ \\
\hline Middle & $136(64.76 \%)$ \\
\hline Upper & $41(19.52 \%)$ \\
\hline
\end{tabular}

\section{TABLE 1: Characteristics of the Study Population}

The most frequent symptom the patient presented with was biliary colic $(n=59,28.1 \%)$, followed by chronic cholecystitis $(n=46,21.9 \%)$ and then acute cholecystitis $(n=38,18.1 \%)($ Table 2$)$. 


\section{Cureus}

Symptoms

Biliary colic

Acute cholecystitis

Chronic cholecystitis

Acute chronic cholecystitis

Acute pancreatitis

Choledocholithiasis

Cholecystoenteric fistula

Biliary ileus n (\%)

$59(28.1 \%)$

$38(18.1 \%)$

$46(21.9 \%)$

$26(12.38 \%)$

$34(16.19 \%)$

$7(3.33 \%)$

$0(0 \%)$

$0(0 \%)$

TABLE 2: Symptoms Presented by the Study Population

Upon assessing the risk factors, $31.84 \%$ of the patients had used oral contraceptives, $32.96 \%$ were pregnant, $20.48 \%$ had diabetes mellitus, and $27.14 \%$ had a history of gallstones. Furthermore, about 114 (54.29\%) patients were overweight, with a body mass index of $25-30 \mathrm{~kg} / \mathrm{m}^{2}$. High triglyceride levels and high cholesterol levels were found in the majority of our study population, which indicated a link between symptomatic gallstones and younger age (Table 3). 


\section{Cureus}

Gallstones

Risk factor

n (\%)

Oral contraceptive usage

Yes

57 (31.84\%)

No

$122(68.16 \%)$

Pregnancy

Yes

$59(32.96 \%)$

No

$120(67.04 \%)$

Diabetes mellitus

Yes

$43(20.48 \%)$

No

167 (79.52\%)

History of gallstones

Yes

$57(27.14 \%)$

No

$153(72.86 \%)$

Socioeconomic status

$33(15.71 \%)$

Lower

$136(64.76 \%)$

Middle

$41(19.52 \%)$

Body mass index $\left(\mathrm{kg} / \mathrm{m}^{2}\right)$

$<25$ (normal)

$74(35.24 \%)$

25-30 (overweight)

$114(54.29 \%)$

$>30$ (obese)

$22(10.48 \%)$

Cholesterol level (mg/dL)

$<200$

95 (45.24\%)

$>200$

$115(54.76 \%)$

Triglyceride level (mg/dL)

$<150$

$102(48.57 \%)$

$>150$

$108(51.43 \%)$

TABLE 3: Distribution of Risk Factors Associated With the Incidence of Gallstones Among the Participants

\section{Discussion}

In our study, we found that young people (mean age: $24.65 \pm 3.25$ years) are more prone to symptomatic gallstones. Gallstones are the most common pathology of the hepatobiliary system with a high prevalence rate among females (80.9\%) as compared with males (19.04\%). Females were more commonly seen with this disease due to a number of factors such as oral contraceptive use and pregnancy. The incidence of this disease in young individuals has increased so rapidly, which in turn costs a lot to the healthcare system, especially in a third-world country such as Pakistan.

A similar study to ours was conducted by Shafique et al., in which $48.13 \%$ of the patients were from an age group below 30 years of age [11]. The study discussed factors such as high body mass index (BMI) and dyslipidemia to be important prognostic factors for gallstones. This was similar to our study, in which 114 
(54.29\%) of the population was overweight, and cholesterol levels greater than $200 \mathrm{mg} / \mathrm{dL}$ were seen in 115 (54.76\%) of the participants. Constantinescu et al. identified patients between the ages of 16 and 25 and found obesity, pregnancy, age, and female gender as important risk factors for the development of gallstones for this age group [12]. The authors also identified that the most commonly seen complications of the disease in young individuals were acute pancreatitis and the presence of stones in the duct. Similarly, Kim et al. studied the young population of Korea, where causes of obesity, such as high BMI, cholesterol, HDL levels, LDL levels, and size of the waist, were risk factors for the development of disease in the young population [13]. These findings were similar to other studies [14,15].

Another study by Sun et al. found that middle- to high-income class individuals was more at risk for developing gallstones [16]. In the present study, individuals belonging to the middle class were more prone to developing gallstones. Sun et al., however, found old age to be a significant contributor to the development of gallstones, not in adolescents below 19 years of age. The elderly were commonly seen with gallstone disease; this was attributed to the aging and sedentary lifestyle of the elderly population. Similarly, Mora-Guzmán et al. revealed that elderly patients were at a higher risk of developing gallstones and gallstone-related complications along with recurrence of gallstones in one-third of patient follow-ups [17].

Overall, our study coincided very well with the current literature. Nevertheless, the study had some limitations. For instance, a larger sample size would have permitted more insight into the factors leading to gallstone formation in the young populations.

\section{Conclusions}

Our study indicated that young people are prone to hepatobiliary issues, with gallstones being the most frequent. The rate of disease was considerably high among females and in patients with high cholesterol and triglyceride levels and abnormal body mass index. Further exploratory studies are needed to determine the cause of gallstones.

\section{Additional Information \\ Disclosures}

Human subjects: Consent was obtained or waived by all participants in this study. Jinnah Postgraduate Medical Centre issued approval F9-98-IRB-2018-G/28867/JPMC. This is to inform you that the study topic entitled "Symptomatic Gallstone in Young Patients Under the Age of 30 Years" has been approved by the committee. Animal subjects: All authors have confirmed that this study did not involve animal subjects or tissue. Conflicts of interest: In compliance with the ICMJE uniform disclosure form, all authors declare the following: Payment/services info: All authors have declared that no financial support was received from any organization for the submitted work. Financial relationships: All authors have declared that they have no financial relationships at present or within the previous three years with any organizations that might have an interest in the submitted work. Other relationships: All authors have declared that there are no other relationships or activities that could appear to have influenced the submitted work.

\section{References}

1. Lammert F, Gurusamy K, Ko CW, et al.: Gallstones. Nat Rev Dis Primers. 2016, 2:16024 10.1038/nrdp.2016.24

2. Radunovic M, Terzic D, Terzic Z, Ratković M, Mugoša B, Andrić B, Radunovic M: Cholecystitis as a cause of abdominal pain in patients with acute viral hepatitis A and B. Acta Med Median. 2012, 51:20-3. 10.5633/amm.2012.0103

3. Di Ciaula A, Wang DQ, Portincasa P: An update on the pathogenesis of cholesterol gallstone disease . Curr Opin Gastroenterol. 2018, 34:71-80. 10.1097/MOG.0000000000000423

4. Kim IS, Myung SJ, Lee SS, Lee SK, Kim MH: Classification and nomenclature of gallstones revisited. Yonsei Med J. 2003, 44:561-70. 10.3349/ymj.2003.44.4.561

5. Alsaif FA, Alabdullatif FS, Aldegaither MK, Alnaeem KA, Alzamil AF, Alabdulkarim NH, Aldohayan AD: Incidence of symptomatic cholelithiasis after laparoscopic sleeve gastrectomy and its association with rapid weight loss. Saudi J Gastroenterol. 2020, 26:94-8. 10.4103/sjg.SJG_472_19

6. Mishra T, Lakshmi KK, Peddi KK: Prevalence of cholelithiasis and choledocholithiasis in morbidly obese South Indian patients and the further development of biliary calculus disease after sleeve gastrectomy, gastric bypass and mini gastric bypass. Obes Surg. 2016, 26:2411-7. 10.1007/s11695-016-2113-4

7. Schirmer BD, Winters KL, Edlich RF: Cholelithiasis and cholecystitis. J Long Term Eff Med Implants. 2005, 15:329-38. 10.1615/jlongtermeffmedimplants.v15.i3.90

8. Schafmayer C, Hartleb J, Tepel J, et al.: Predictors of gallstone composition in 1025 symptomatic gallstones from Northern Germany. BMC Gastroenterol. 2006, 6:36. 10.1186/1471-230X-6-36

9. Gutt C, Schläfer S, Lammert F: The treatment of gallstone disease . Dtsch Arztebl Int. 2020, 117:148-58. 10.3238/arztebl.2020.0148

10. Bortoff GA, Chen MY, Ott DJ, Wolfman NT, Routh WD: Gallbladder stones: imaging and intervention. Radiographics. 2000, 20:751-66. 10.1148/radiographics.20.3.g00ma16751

11. Shafique MS, Ahmad R, Ahmad SH, Hassan SW, Khan JS: Gallstones in young population and its complications. Ulutas Med J. 2018, 4:131-8. 10.5455/umj.20180324011035

12. Constantinescu T, Huwood Al Jabouri AK, Brãtucu E, Olteanu C, Toma M, Stoiculescu A: Gallstone disease in 


\section{Cureus}

young population: incidence, complications, therapeutic approach. Chirurgia. 2012, 107:579-82.

13. Kim HS, Cho SK, Kim CS, Park JS: Big data and analysis of risk factors for gallbladder disease in the young generation of Korea. PLoS One. 2019, 14:e0211480. 10.1371/journal.pone.0211480

14. Mewa Kinoo S, Nagiah S, Chuturgoon A, Singh B: Symptomatic gallstones and HIV in black South African women: changing trends of gallstone disease?. South Afr J HIV Med. 2021, 22:1208.

10.4102/sajhivmed.v22i1.1208

15. Alishi YA, Howaish FA, Alhamdan FA, et al.: Prevalence and risk factors for gallstones among population in Riyadh City, KSA 2017. Egypt J Hosp Med. 2017, 69:2384-8. 10.12816/0041681

16. Sun H, Tang H, Jiang S, Zeng L, Chen EQ, Zhou TY, Wang YJ: Gender and metabolic differences of gallstone diseases. World J Gastroenterol. 2009, 15:1886-91. 10.3748/wig.15.1886

17. Mora-Guzmán I, Di Martino M, Bonito AC, Jodra VV, Hernández SG, Martin-Perez E: Conservative management of gallstone disease in the elderly population: outcomes and recurrence. Scand J Surg. 2020, 109:205-10. 10.1177/1457496919832147 